\title{
The IntSat method for Integer Linear Programming
}

\author{
Robert Nieuwenhuis \\ Barcelogic.com and Technical University of Catalonia (UPC), Barcelona
}

\begin{abstract}
Conflict-Driven Clause-Learning (CDCL) SAT solvers can automatically solve very large real-world problems. To go beyond, and in particular in order to solve and optimize problems involving linear arithmetic constraints, here we introduce IntSat, a generalization of CDCL to Integer Linear Programming (ILP). Our simple 1400-line C++ prototype IntSat implementation already shows some competitiveness with commercial solvers such as CPLEX or Gurobi. Here we describe this new IntSat ILP solving method, show how it can be implemented efficiently, and discuss a large list of possible enhancements and extensions.
\end{abstract}

\section{Introduction}

Conflict-Driven Clause-Learning (CDCL) propositional SAT solving technology can automatically solve hard real-world industrial and scientific problem instances involving large numbers of binary variables and constraints (i.e., clauses). SAT is the particular case of ILP where all variables are binary (0/1) and constraints have the form $x_{1}+\ldots+x_{m}-y_{1} \ldots-y_{n}>-n$, written as clauses $x_{1} \vee \ldots \vee x_{m} \vee \overline{y_{1}} \vee \ldots \vee \overline{y_{n}}$, i.e., sets (disjunctions) of literals. Given a partial assignment $A$, seen as a set of (non-contradictory) literals, a clause $C$ is true in $A$ if $A \cap C \neq \emptyset$, it is false or a conflict if $\bar{l} \in A$ for every literal $l$ in $C$, and it is undefined otherwise. All essential CDCL features are described in the following 14-line algorithm, where $A$ is seen as an (initially empty) stack:

1. Propagate: while possible and no conflict appears, if, for some clause $l \vee C, C$ is false in $A$ and $l$ is undefined, push $l$ onto $A$, associating to $l$ the reason clause $C$.

2. if there is no conflict

if all variables are defined in $A$, output "solution $A$ " and halt.

else Decide: push some undefined literal $l$, marked as a decision, and goto step 1 .

3. If $A$ contains no decisions, output "unsatisfiable" and halt.

4. Use a clause data structure $C$. Initially, let $C$ be any conflict.

- Conflict analysis: Invariant: $C$ is false in $A$, that is, if $l \in C$ then $\bar{l} \in A$. If $l$ is the literal of $C$ whose negation is topmost in $A$, and $D$ is the reason clause of $\bar{l}$, then replace $C$ by $(C \backslash\{l\}) \cup D$. Repeat this until there is only one literal $l_{\text {top }}$ in $C$ such that $\overline{l_{\text {top }}}$ is, or is above, $A$ 's topmost decision.

- Backjump: pop literals from $A$ until either there are no decisions in $A$ or, for some $l$ in $C$ with $l \neq l_{\text {top }}$, there are no decisions above $\bar{l}$ in $A$.

- Learn: add the final $C$ as a new clause, and go to 1 (where $C$ propagates $l_{\text {top }}$ ).

Note that replacing $C$ by $(C \backslash\{l\}) \cup D$ is in fact an inference by resolution between $C$ and $D \vee \bar{l}$. Essentially all state-of-the-art CDCL SAT solvers use this (so called IUIP conflict analysis-based) algorithm, with efficient data structures for propagation, heuristics that select recently active literals as decisions, periodically forgetting (removing) the least useful learned clauses, and using clause simplification and other inprocessing methods (see, e.g., $[16,4]$ for more details and further references). 
Given CDCL's enormous success for SAT, for decades researchers (e.g., from the SAT community, but not only) have tried to produce an effective CDCL-like method for ILP. However, due to a number of obstacles (see, e.g., Example 3 below), the results of such attempts were always orders of magnitude slower than the state-of-the-art commercial MIP/ILP solvers such as CPLEX or Gurobi, based on LP relaxations, simplex, and branch-and-cut (see, e.g., the "mip basics" at www . gurobi .com). Typical attempts to generalize CDCL from SAT to ILP are in the following sense, where (possibly subindexed) letters $a$ denote integer coefficients:

\begin{tabular}{|cc|cc|}
\hline SAT & & ILP \\
\hline clause & $l_{1} \vee \ldots \vee l_{n}$ & linear constraint & $a_{1} x_{1}+\cdots+a_{n} x_{n} \leq a_{0}$ \\
$0 / 1$ variable & $x$ & integer variable & $x$ \\
positive literal & $x$ & lower bound & $a \leq x$ \\
negative literal & $\bar{x}$ & upper bound & $x \leq a$ \\
propagation & & bound propagation & \\
resolution inference & & cut inference & \\
\hline
\end{tabular}

Example 1. By bound propagation, from the lower bound $1 \leq x$, the upper bound $y \leq 2$, and the constraint $x-2 y+5 z \leq 5$, we infer that $1-4+5 z \leq 5$, so $5 z \leq 8$, and hence $z \leq 8 / 5$, which is rounded, propagating a new bound $z \leq 1$. Note that any 1 -variable constraint propagates a bound by itself, e.g., from $-7 x \leq 3$ we have $-3 \leq 7 x$, and hence $-3 / 7 \leq x$, which after rounding propagates the lower bound $0 \leq x$.

Example 2. From $4 x+4 y+2 z \leq 3$ and $-10 x+y-z \leq 0$, by multiplying the former by 5 and the latter by 2 and adding them up, we obtain the cut $22 y+8 z \leq 15$. Here the variable $x$ is eliminated, which can always be achieved if in the two premises $x$ has coefficients $a$ and $b$ such that $a \cdot b<0$. The result $22 y+8 z \leq 15$ can be normalized dividing by $\operatorname{gcd}(22,8)=2$, giving $11 y+4 z \leq 15 / 2$ and, by rounding, $11 y+4 z \leq 7$.

Example 3. An important problem for extending CDCL to ILP is the following, which we will call the rounding problem. Assume we have the two constraints $x+y+2 z \leq 2$ and $x+y-2 z \leq 0$ and we take the decision $0 \leq x$, which propagates nothing, and later on another decision $1 \leq y$, which due to $x+y+2 z \leq 2$ propagates $z \leq 0$ (since $1+2 z \leq 2$, and hence $2 z \leq 1$ and by rounding $z \leq 1 / 2$ we get $z \leq 0)$. Then $x+y-2 z \leq 0$ becomes a conflict: it is false in the current partial assignment $A=\{0 \leq x, 1 \leq y, z \leq 0\}$.

Now let us attempt a straightforward generalization of the CDCL algorithm: since $z \leq 0$ is the topmost (last propagated) bound, a cut inference eliminating $z$ between both constraints would be needed, generating the new constraint $C$ which is $2 x+2 y \leq 2$, or equivalently, $x+y \leq 1$. Then conflict analysis is over because there is only one bound in $A$ at, or above, the last decision relevant for $C$, namely $1 \leq y$. But unfortunately the new constraint $x+y \leq 1$ is not false in $A$, breaking (what should be) the invariant. Hence it does not propagate the negation of $1 \leq y$ (which is $y \leq 0$ ) and is too weak to force a backjump. This problem is due to the rounding that takes place when propagating $z$.

The rounding problem illustrated in Example 3 was solved in a very ingenious way by Jovanovic and de Moura in their cutsat procedure [14], where a decision can only 
make a variable equal to its current upper or lower bound, which permits, at each conflict caused by bound propagations with rounding, to compute tightly propagating constraints that justify the same propagations without rounding, and to do conflict analysis using tightly propagating constraints only. This makes the learning scheme of [14] similar to the SAT one of doing resolutions until reaching a clause built from decisions only, which is well known to perform significantly worse than 1UIP.

Here we introduce IntSat, a new completely different method for ILP. It admits arbitrary new bounds as decisions and guides the search exactly as with the 1UIP approach in CDCL-based SAT solving, and still it overcomes the rounding problem. The key ideas behind IntSat are as follows. Given a partial assignment $A$, a set (stack) of bounds, each time a constraint $C$ and a set of bounds $R$ with $R \subseteq A$ propagate a new bound $B$, this bound is pushed onto $A$, associating to $B$ not only its reason constraint $C$ but also its reason set $R$. Conflict analysis and cuts performed are both guided by successive refinements of a so-called Conflicting Set of bounds $C S \subseteq A$ that is infeasible along with the current set of constraints. After each conflict, always a backjump takes place and a new constraint is learned.

This paper is structured as follows. The basic IntSat procedure is introduced in Section 2, its termination, correctness and completeness stated, and extensions are given for, e.g., optimization. In Section 3 we give some details about the efficient implementability of IntSat, and further work on IntSat is discussed in Section 4. Section 5 provides experimental results and in Section 6 we describe related work and conclude.

\section{The basic IntSat procedure}

In a first basic version of IntSat we deal with integer coefficients only and decide the existence of integer solutions, i.e., feasibility problems only, and no optimization yet (extensions are handled later on). Let $X$ be a finite set of variables $\left\{x_{1} \ldots x_{n}\right\}$. An (integer linear) constraint over $X$ is an expression of the form $a_{1} x_{1}+\cdots+a_{n} x_{n} \leq a_{0}$ where, for all $i$ in $0 \ldots n$, the coefficients $a_{i}$ are integers. Below, variables are always denoted by (possibly sub-indexed or primed) lowercase $x, y, z$ and coefficients by $a, b, c$, respectively. An Integer Program (IP) over $X$ is a set $S$ of integer linear constraints over $X$. A solution for an IP $S$ over $X$ is a function sol: $X \rightarrow \mathbb{Z}$ that satisfies every constraint $a_{1} x_{1}+\cdots+a_{n} x_{n} \leq a_{0}$ in $S$, that is, $a_{1} \cdot \operatorname{sol}\left(x_{1}\right)+\cdots+a_{n} \cdot \operatorname{sol}\left(x_{n}\right) \leq_{\mathbb{Z}} a_{0}$. A bound is a one-variable constraint $a_{1} x \leq a_{0}$. Any bound can equivalently either be written as a lower bound $a \leq x$ or as an upper bound $x \leq a$. For a constraint $a_{1} x_{1}+\ldots+a_{n} x_{n} \leq a_{0}$, each $a_{i} x_{i}$ is called a monomial of it, and the monomial is positive (negative) if $a_{i}$ is.

Bound propagation: Let $C$ be a constraint of the form $P+a x \leq a_{0}$, where $P$ is a sum of positive monomials $\left\{b_{1} y_{1}, \ldots, b_{p} y_{p}\right\}$ and negative monomials $\left\{c_{1} z_{1}, \ldots, c_{q} z_{q}\right\}$. Let $R$ be a set of bounds $\left\{l b_{1} \leq y_{1}, \ldots, l b_{p} \leq y_{p}, z_{1} \leq u b_{1}, \ldots, z_{q} \leq u b_{q}\right\}$. Let $E$ denote the expression $a_{0}-b_{1} l b_{1}-\ldots-b_{p} l b_{p}-c_{1} u b_{1}-\ldots-c_{q} u b_{q}$. If $a<0$ then then $C$ and $R$ propagate $\lceil E / a\rceil \leq x$. If $a>0$ then then $C$ and $R$ propagate $x \leq\lfloor E / a\rfloor$.

Cuts and constraint normalization: In what follows we assume all constraints to be eagerly normalized: any constraint $a_{1} x_{1}+\ldots+a_{n} x_{n} \leq a_{0}$ with $d=\operatorname{gcd}\left(a_{1}, \ldots, a_{n}\right)>1$ is eagerly replaced by $a_{1} / d \quad x_{1}+\ldots+a_{n} / d \quad x_{n} \leq\left\lfloor a_{0} / d\right\rfloor$. From constraints 
$a_{1} x_{1}+\cdots+a_{n} x_{n} \leq a_{0}$ and $b_{1} x_{1}+\cdots+b_{n} x_{n} \leq b_{0}$, and natural numbers $c$ and $d$, a new constraint $c_{1} x_{1}+\cdots+c_{n} x_{n} \leq c_{0}$ called a $c u t$ can be obtained where $c_{i}=c a_{i}+d b_{i}$ for $i$ in $0 \ldots n$. If some $c_{i}=0$ then we say that $x_{i}$ is eliminated in this cut. Note that if $a_{i} b_{i}<0$, then one can always choose $c$ and $d$ such that $x_{i}$ is eliminated. See [13, 7, 23] for further discussions and references about Chvátal-Gomory cuts and their applications to ILP.

Let $A$ be a set of bounds. A variable $x$ is defined to $a$ in $A$ if $\{a \leq x, x \leq a\} \subseteq A$ for some $a$. We call two bounds $a \leq x$ and $x \leq a^{\prime}$ contradictory if $a>a^{\prime}$. Note that if all variables of $X$ are defined and there are no contradictory bounds in $A$ then $A$ can be seen as a total assignment $A: X \rightarrow \mathbb{Z}$. A bound $a \leq x$ is new in $A$ if there is no $a^{\prime} \leq x$ in $A$ with $a^{\prime} \geq a$. Similarly, $x \leq a$ is new if there is no $x \leq a^{\prime}$ with $a^{\prime} \leq a$. A bound is fresh in $A$ if it is new in $A$ and contradictory with no bound in $A$.

False constraint (conflict) in $A$ : If $C$ is a constraint $a_{1} x_{1}+\ldots+a_{n} x_{n} \leq a_{0}$ with positive monomials $\left\{b_{1} y_{1}, \ldots, b_{p} y_{p}\right\}$, negative monomials $\left\{c_{1} z_{1}, \ldots, c_{q} z_{q}\right\}$ and there is a subset of bounds $\left\{l b_{1} \leq y_{1}, \ldots, l b_{p} \leq y_{p}, \quad z_{1} \leq u b_{1}, \ldots, z_{q} \leq u b_{q}\right\} \subseteq A$ with $b_{1} l b_{1}+\ldots+b_{p} l b_{p}+$ $c_{1} u b_{1}+\ldots+c_{q} u b_{q}>a_{0}$ then $C$ is false or a conflict in $A$.

The basic IntSat algorithm: In the following IntSat algorithm, $A$ is seen as an (initially empty) stack of bounds:

1. Propagate: while possible and no conflict appears, if $C$ and $R$ propagate some fresh bound $B$, for some constraint $C$ and set of bounds $R$ with $R \subseteq A$, then push $B$ onto $A$, associating to $B$ the reason constraint $C$ and the reason set $R$.

2. if there is no conflict

if all variables are defined in $A$, output "solution $A$ " and halt.

else Decide: push some fresh bound $B$, marked as a decision, and go to 1 .

3. If $A$ contains no decisions, output "infeasible" and halt.

4. Use data structures $C$, a constraint, and $C S$, the Conflicting Set of bounds. Initially, $C$ is any conflict and $C S$ is the subset of bounds of $A$ causing the falsehood of $C$.

- Conflict analysis: Invariants: $C S \subseteq A$ and if $S$ is the current set of constraints, then $S \cup C S$ is infeasible (has no solution). Repeat the following three steps:

- If $B$ is the bound in $C S$ that is topmost in $A$, and $R$ is the reason set of $B$, then let $C S$ be $(C S \backslash\{B\}) \cup R$.

- If a cut eliminating $B$ 's variable exists between $C$ and $B$ 's reason constraint then replace $C$ by that cut.

- Early Backjump: If for some maximal $k \in \mathbb{N}$, after popping $k$ bounds the last one being a decision, $C$ propagates some new bound in the resulting $A$, then pop $k$ bounds, learn $C$ as a new constraint, and go to 1 .

until $C S$ contains a single bound $B_{\text {top }}$ that is, or is above, $A$ 's topmost decision.

- Backjump: Pop bounds from $A$ until either there are no decisions in $A$ or, for some $B$ in $C S$ with $B \neq B_{\text {top }}$, there are no decisions above $B$ in $A$. Then push $\overline{B_{\text {top }}}$ with associated reason constraint $C$ and reason set $C S \backslash\left\{B_{\text {top }}\right\}$.

- Learn: add the final $C$ as a new constraint, and go to 1.

Note that in the 2nd step of conflict analysis indeed sometimes no cut eliminating $B$ 's variable exists between $C$ and $B$ 's reason constraint; this can be because that variable does not occur in $C$, or it occurs with the same sign. 
Example 4. We apply IntSat to Example 3: there are two constraints $x+y+2 z \leq 2$ and $x+y-2 z \leq 0$ and we take the decision $0 \leq x$, which propagates nothing, and later on another decision $1 \leq y$, which due to $x+y+2 z \leq 2$ propagates $z \leq 0$, which is pushed with associated reason constraint $x+y+2 z \leq 2$ and reason set $\{0 \leq x, 1 \leq y\}$. Conflict analysis: Initially, $x+y-2 z \leq 0$ is the conflict $C$ and $C S$ is the set of bounds $\{0 \leq x, 1 \leq y, z \leq 0\} \subseteq A$ causing the falsehood $C$. In the first iteration, in $C S$ we replace $z \leq 0$ by its reason set $\{0 \leq x, 1 \leq y\}$. The resulting $C S$ is $\{0 \leq x, 1 \leq y\}$. A cut eliminating $z$ exists (see Example 3 ) and $C$ becomes $x+y \leq 1$. Then conflict analysis is over because the $C S$ contains exactly one bound $B_{\text {top }}$, which is $1 \leq y$, at or above $A$ 's topmost decision. Backjump: We pop bounds until for some $B$ in $C S$ with $B \neq B_{\text {top }}$, there are no decisions above $B$ in $A$, in this case, until there are no decisions above $0 \leq x$ in $A$, and then push $\overline{B_{t o p}}$, which is $y \leq 0$, with reason set $\{0 \leq x\}$, and with reason constraint $x+y \leq 1$. Note that this reason constraint is not a "good" reason, i.e., it does not propagate $y \leq 0$, but still $y \leq 0$ is a valid consequence of the set of constraints together with its reason set $\{0 \leq x\}$. Learn: The final $C$, which is $x+y \leq 1$, is learned.

Example 5. Consider the initial constraints

$$
\begin{array}{lr}
C_{0}: & x-3 y-3 z \leq 1 \\
C_{1}: & -2 x+3 y+2 z \leq-2 \\
C_{2}: & 3 x-3 y+2 z \leq-1
\end{array}
$$

Below we depict the stack with some initial bounds after doing their propagations and taking and propagating two decisions:

\begin{tabular}{|c|c|c|} 
& & \\
$2 \leq y$ & $\{1 \leq x, z \leq-2\}$ & $C_{0}$ \\
\hline$x \leq 1$ & $\{y \leq 2, z \leq-2\}$ & $C_{0}$ \\
\hline$z \leq-2$ & \multicolumn{2}{|c|}{ decision } \\
\hline$z \leq-1$ & $\{x \leq 2,1 \leq y\}$ & $C_{1}$ \\
\hline$x \leq 2$ & \multicolumn{2}{|c|}{ decision } \\
\hline$z \leq 0$ & $\{x \leq 3,1 \leq y\}$ & $C_{1}$ \\
\hline$y \leq 2$ & $\{x \leq 3,-2 \leq z\}$ & $C_{1}$ \\
\hline $1 \leq x$ & $\{1 \leq y,-2 \leq z\}$ & $C_{1}$ \\
\hline$z \leq 2$ & \multicolumn{2}{|c|}{ initial } \\
\hline$-2 \leq z$ & \multicolumn{2}{|c|}{ initial } \\
\hline$y \leq 4$ & \multicolumn{2}{|c|}{ initial } \\
\hline $1 \leq y$ & \multicolumn{2}{|c|}{ initial } \\
\hline$x \leq 3$ & reason set reason constraint \\
\hline$-2 \leq x$ & renl \\
\hline bound
\end{tabular}

Now $C_{1}$ is a conflict. The initial $C S$ is $\{-2 \leq z, x \leq 1,2 \leq y\}$, with two bounds above the last decision. In the first conflict analysis step, we replace $2 \leq y$ by its reason set $\{1 \leq x, z \leq-2\}$ obtaining the new $C S\{-2 \leq z, 1 \leq x, z \leq-2, x \leq 1\}$ which still has two bounds at or above the last decision. Now a cut eliminating $y$ is attempted between 
the initial $C$, which is $C_{1}$, and the reason constraint of $2 \leq y$, which is $C_{0}$. Here this cut exists, with $c=d=1$, and we obtain and learn the new constraint $C_{3}:-x-z \leq-1$. It allows us to perform an early backjump to before the second decision, since there it propagates $2 \leq x$ with reason set $\{z \leq-1\}$ and reason constraint $C_{3}$. Then, after two more propagations, we obtain

\begin{tabular}{|c|c|c|} 
& & \\
$2 \leq y$ & $\{2 \leq x, z \leq-1\}$ & $C_{0}$ \\
\hline$-1 \leq z$ & $\{x \leq 2\}$ & $C_{3}$ \\
\hline $2 \leq x$ & $\{z \leq-1\}$ & $C_{3}$ \\
\hline$z \leq-1$ & $\{x \leq 2,1 \leq y\}$ & $C_{1}$ \\
\hline$x \leq 2$ & \multicolumn{2}{|c|}{ decision } \\
\hline$z \leq 0$ & $\{x \leq 3,1 \leq y\}$ & $C_{1}$ \\
\hline$y \leq 2$ & $\{x \leq 3,-2 \leq z\}$ & $C_{1}$ \\
\hline $1 \leq x$ & $\{1 \leq y,-2 \leq z\}$ & $C_{1}$ \\
\hline$z \leq 2$ & \multicolumn{2}{|c|}{ initial } \\
\hline$\ldots$ & $\ldots$ \\
\hline$-2 \leq x$ & initial \\
\hline
\end{tabular}

and again $C_{1}$ is a conflict, with the initial $C S$ being $\{x \leq 2,-1 \leq z, 2 \leq y\}$. After the first conflict analysis step (replacing $2 \leq y$ ) the $C S$ becomes $\{x \leq 2, z \leq-1,2 \leq$ $x,-1 \leq z\}$. As before, the cut eliminates $y$, between $C_{1}$ and $C_{0}$ (the initial $C$ and the reason constraint of $2 \leq y$ ), obtaining $-x-z \leq-1$. After the following step (replacing $-1 \leq z$ ), the $C S$ becomes $\{x \leq 2, z \leq-1,2 \leq x\}$. The $C$ does not change because no cut eliminating $z$ exists with $C_{3}$. In the next step (replacing $2 \leq x$ ), the $C S$ becomes $\{x \leq 2, z \leq-1\}$. Again no cut eliminating $z$ exists with $C_{3}$. In another step (replacing $z \leq-1)$, the $C S$ becomes $\{1 \leq y, x \leq 2\}$. Since there is only one bound at or after the last decision, we backjump, in this case to before the first decision, and add there the negation of $x \leq 2$, which is $3 \leq x$.

The result of the cut on $C$ with $C_{1}$ eliminating $z$ gives us $-4 x+3 y \leq-4$. The backjump with this cut $\left(C_{4}\right)$ can also take us to before the first decision, but propagating $2 \leq x$. Since this is weaker than the bound $3 \leq x$ obtained from the $C S$, here we choose the $C S$ one. After one further propagation, the procedure returns "infeasible" since the conflict $C_{2}$ appears and there are no decisions in the stack:

\begin{tabular}{|c|c|c|} 
& & \\
\hline $3 \leq x$ & $\{3 \leq x, y \leq 2\}$ & $C_{0}$ \\
\hline$z \leq 0$ & $\{x \leq 3,1 \leq y\}$ & $C_{4}$ \\
\hline$y \leq 2$ & $\{x \leq 3,-2 \leq z\}$ & $C_{1}$ \\
\hline $1 \leq x$ & $\{1 \leq y,-2 \leq z\}$ & $C_{1}$ \\
\hline$z \leq 2$ & \multicolumn{2}{|c|}{ initial } \\
\hline$\ldots$ & $\ldots$ \\
\hline$-2 \leq x$ & initial \\
\hline
\end{tabular}


Theorem 1. The basic IntSat algorithm, when given as input a finite set of constraints $S$ including for each variable $x_{i}$ a lower bound $l b_{i} \leq x_{i}$ and an upper bound $x_{i} \leq u b_{i}$, always terminates, finding a solution if, and only if, there exists one, and returning "infeasible" if, and only if, $S$ is infeasible.

The previous theorem holds even if no cuts are performed and no new constraints are learned (although practical performance depends crucially on these). Its proof follows essentially the same scheme as our termination, soundness and completeness results for SAT and SAT Modulo Theories (SMT) [20]. For termination (from which soundness and completeness are not hard to establish), we define a well-founded ordering $>$ on the states of the stack $A$, as follows. For a given $A$, the number of possible values a variable $x_{i}$ can still take is $v_{i}(A)=u b_{i}-l b_{i}+1$, where $l b_{i} \leq x_{i}$ and $x_{i} \leq u b_{i}$ are its topmost lower and upper bounds in $A$, and the total number of values for all $n$ variables is $v(A)=v_{1}(A)+\ldots v_{n}(A)$. Let $A_{i}$, for $i \geq 1$ denote the bottom part of $A$, below (and without) the $i$-th decision. We define a stack $A$ to be larger (i.e., less advanced, searchwise) than a stack $A^{\prime}$, written $A>A^{\prime}$, if $\left\langle v\left(A_{1}\right), \ldots, v\left(A_{m}\right)\right\rangle>_{l e x}\left\langle v\left(A_{1}^{\prime}\right), \ldots, v\left(A_{m}^{\prime}\right)\right\rangle$ where $m$ is the maximal number of decisions the stack can contain, at most $n \cdot v(A)$ for the initial $A$. It is easy to see that this lexicographic ordering $>$ is well-founded and that all steps of the algorithm either halt it or transform $A$ into an $A^{\prime}$ with $A>A^{\prime}$.

More general constraints: It is obvious that a constraint $a_{1} x_{1}+\ldots+a_{n} x_{n} \geq a_{0}$ can be expressed as $-a_{1} x_{1}-\ldots-a_{n} x_{n} \leq-a_{0}$, that $a_{1} x_{1}+\ldots+a_{n} x_{n}=a_{0}$ can be replaced by the two constraints $a_{1} x_{1}+\ldots+a_{n} x_{n} \leq a_{0}$ and $a_{1} x_{1}+\ldots+a_{n} x_{n} \geq a_{0}$, and that rational non-integer coefficients $a / b$ can be removed by multiplying the constraint by $b$.

Optimization is also possible in a standard way, since, unlike what happens in SAT, linear constraints are first-class citizens (i.e., belong to the core language). For finding a solution that minimizes a linear expression $a_{1} x_{1}+\ldots+a_{n} x_{n}$ (or maximizes $-a_{1} x_{1}-\ldots-$ $a_{n} x_{n}$ ), in our current implementation this is done in a completely straightforward way: first an arbitrary solution $A$ is found and then, each time a new solution $A$ is found, it is attempted to improve it by re-running with the additional constraint $a_{1} x_{1}+\ldots+a_{n} x_{n} \leq$ $a_{0}$ where $a_{0}$ is $a_{1} A\left(x_{1}\right)+\ldots+a_{n} A\left(x_{n}\right)-1$. This is done until the problem becomes infeasible. Bound propagations from these successively stronger constraints are indeed very effective for pruning (bounding) the resulting branch-and-bound search.

Handling unbounded variables. Up to now we have assumed that for each variable there is an initial lower bound and an upper bound, or, equivalently, initial constraints propagating such bounds. Although this is common in practical applications, some problems do have unbounded variables. In theory, any ILP can be converted into an equivalent fully bounded one [23], but these bounds are too large to be useful in practice. One solution is to introduce a fresh auxiliary variable $z$, with lower bound $0 \leq z$, and for each variable $x$ without lower bound add the constraint $-z \leq x$, and similarly if it has no upper bound add $x \leq z$. Then one can re-run the IntSat procedure with successively larger upper bounds $z \leq u b$ for $z$, thus guaranteeing completeness for finding (optimal) solutions. Further practical solutions are subject of current work, also for handling the well-known fact that with unbounded variables bound propagation may not terminate in unfeasible problems: consider, e.g., $C_{1}: x-y \leq 0$ and $C_{2}:-x+y+1 \leq 0$ and the bound $0 \leq x$, which makes $C_{1}$ propagate $0 \leq y$; then $C_{2}$ propagates $1 \leq x$, and so on. 


\section{Implementation}

Here we describe some details of our current prototype IntSat implementation. It currently consists of 1400 lines of simple $\mathrm{C}++$ code that make heavy use of standard STL data structures (this source code can be downloaded from [19]). For instance, a constraint is an STL vector of monomials (pairs of two ints: the variable number and the coefficient), sorted by variable number, plus some additional information (independent term, activity). Coefficients are never larger than $2^{30}$, and cuts producing any coefficient larger than $2^{30}$ are simply not performed, which is a straightforward way of guaranteeing that no overflow occurs if bound propagation, cuts, normalization, etc., are done using 64-bit integers for intermediate results. During conflict analysis, the $C S$ is implemented simply as an STL set of ints, the heights in the stack of the bounds in the CS. A very large source of inefficiency of conflict analysis is our current implementation of Early Backjumps, which, after each cut giving a new $C$, naively checks, at all (frequently thousands of) prefixes of the stack below a decision, whether $C$ propagates any new bound at that prefix.

The current assignment There is an array, the Bounds Array, indexed by variable number, that can return in constant time the current upper and lower bounds for that variable. It always stores, for each variable $x_{i}$, the positions $p l_{i}$ and $p u_{i}$ in the stack of its current (strongest) upper bound and lower bound, respectively, with $p l_{i}=0\left(p u_{i}=0\right)$ if $x_{i}$ has no current lower (upper) bound. The stack itself is another array containing at each position three data fields: a bound, a natural number pos, and an info field containing, among other information, (pointers to) the reason set and the reason constraint. The value pos is always the position in the stack of the previous bound of the same type (lower or upper) for this variable, with pos $=0$ for initial bounds. When pushing or popping bounds, these properties are easy to maintain in constant time.
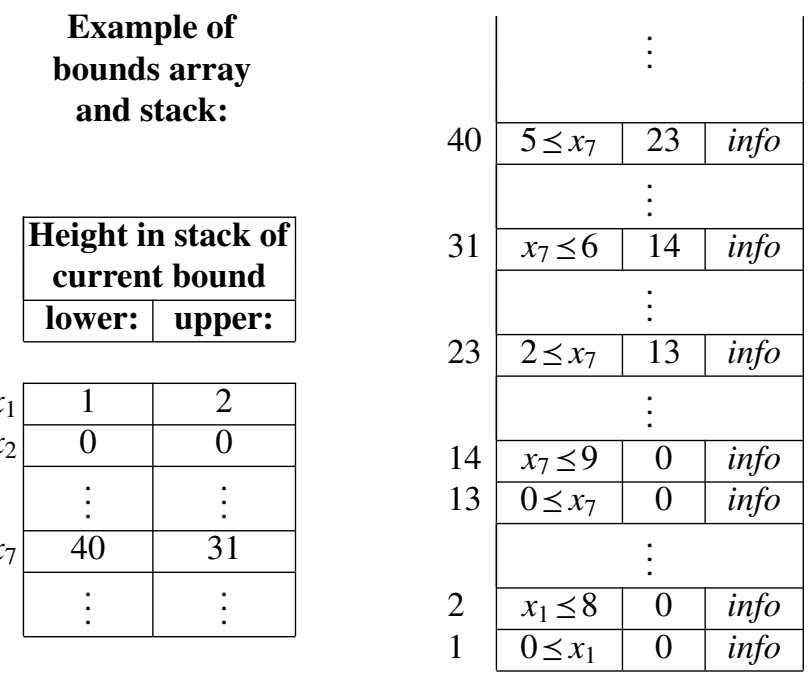

Bound propagation using filters: Affordably efficient bound propagation is crucial for performance. In our current implementation, for each variable $x$, there are two occurs 
lists. The positive occurs list for $x$ contains all pairs $\left(I_{C}, a\right)$ s.t. $C$ is a linear constraint where $x$ occurs with positive coefficient $a$, and the negative one contains the same for occurrences with a negative coefficient $a$. Here $I_{C}$ is an index to the constraint header of $C$ in an array of constraint headers. Each constraint header contains an integer $F_{C}$ called a filter, and (a pointer to) the constraint $C$ itself. The filter $F_{C}$ is maintained cheaply, and one can guarantee that $C$ does not propagate anything as long as $F_{C} \leq 0$, thus avoiding many useless (cache-) expensive visits to the actual constraint $C$. This is done as follows.

Let $C$ be a constraint of the form $a_{1} x_{1}+\cdots+a_{n} x_{n} \leq a_{0}$. Let $l b_{i} \leq x_{i}$ and $x_{i} \leq u b_{i}$ be the current lower and upper bounds (if any) for $x_{i}$. Each monomial $a_{i} x_{i}$ in $C$ can have a minimal value $m_{i}$, which is $a_{i} \cdot l b_{i}$ if $a_{i} \geq 0$, and $a_{i} \cdot u b_{i}$ otherwise. Here $m_{i}$ is undefined if there is no such bound $l b_{i}$ (or $u b_{i}$ ). Initially, if some $m_{i}$ is undefined, then $F_{C}$ is set to a special value $\perp$, and otherwise to $-a_{0}+m_{1}+\cdots+m_{n}+\max _{i}\left\{\left|a_{i}\left(u b_{i}-l b_{i}\right)\right|\right\}$. In the latter case, $F_{C}$ is said to be precise: the constraint $C$ propagates if, and only if, $\perp \neq F_{C}>0$. At all time points, $F_{C}=\perp$ or $F_{C}$ is an upper approximation of the precise one, so $C$ can only propagate (or be false) if $F_{C}>0$.

To preserve this property, these filters need to be updated when new bounds are pushed onto the stack (and each update needs to be undone when popped, for which other data structures exist). Assume a new lower bound $k \leq x$ is pushed onto the stack. Let the previous lower bound for $x$ (if any) be $k^{\prime} \leq x$. For each pair $\left(I_{C}, a\right)$ in the positive occurs list of $x$, using $I_{C}$ we access the $F_{C}$ and increase it by $\left|a\left(k-k^{\prime}\right)\right|$. If there was no previous lower bound, then $F_{C}$ was $\perp$ and is now set to 1 . If $F_{C}$ becomes positive, the constraint $C$ is visited because it may propagate some new bound. After each time a constraint $C$ is visited, $F_{C}$ is set to its precise value. If a new upper bound $x \leq k$ is pushed on the stack, exactly the same is done, where $x \leq k^{\prime}$ is the previous upper bound for $x$ (if any), and using the negative occurs list.

\section{Further work}

Both from a theoretical as a practical point of view, a large amount of further ideas around IntSat arise to be explored. From the implementation point of view, aspects such as special treatments for binary variables and for specific types of constraints should be worked out. Our current implementation in fact mimics several ideas from CDCL SAT solving without having tested them thoroughly.

Decision heuristics. For instance, our current heuristics for selecting the variable of the next decision bound are based on recent activity: the variable with the highest activity score is picked (for this there is a priority queue). The activity score of a variable $x$ is increased each time a bound containing $x$ appears in the $C S$ during conflict analysis, and to reward recent activity the amount of increment grows in time. Once a variable is picked, one has to decide the actual decision bound: whether it is lower or upper, and how to divide the interval between the current lower and upper bounds. Another idea is to try to mimic the last-phase polarity heuristic from SAT [22], which would translate into picking some recent upper/lower bound and value for the selected variable. 
Restarts and cleanups. Something similar happens with the periodic restarts that SAT solvers apply. We currently follow a rather conservative restart policy based on increasing intervals based on the number of conflicts. Another basically non-tested aspect is the cleanup policy for the constraint database; at each cleanup, we remove all non-initial constraints with more than two monomials and activity counter equal to 0 . This activity counter is increased each time the constraint is a conflicting or reason constraint at conflict analysis, and is divided by 2 at each cleanup. Cleanups are done periodically, in such a way that the constraint database grows rather slowly over time.

Early backjump and conflict analysis. Performing early backjumps is in fact optional. When omitted (or not done whenever possible), the price to be paid is the loss of an invariant of the stack: it is no longer true that before each decision all bounds are exhaustively propagated. A slight modification of conflict analysis suffices to handle this: before starting conflict analysis, pop bounds from the stack until the initial $C S$ contains at least one bound at or above the topmost decision. We have not done any thorough experiments yet evaluating this option. One could, for example, do, or attempt to do, early backjump with the intermediate conflicting constraint $C$ if it is false in the current stack, or only if it is promising (e.g., short) according to some heuristic. In any case, further work on the implementation should probably cover a much better implementation for early backjumping, which is currently a black hole for efficiency.

Several other improvements exist for conflict analysis. For example, the quality of backjumps and the strength of the reason sets can be improved by doing some more work: the $C S$ can be simplified by removing bounds that are subsumed by stronger ones, and also, instead of using the pre-stored reason sets $R$, one can re-compute them on the fly during conflict analysis with similar aims. One can also do a bit of search during conflict analysis, e.g., by trying to remove non-topmost bounds and do cuts with these, with the aim of finding good early backjump cuts.

Optimization. For optimization many further ideas exist: heuristics for finding a first solution quickly (which helps bounding the search dramatically), heuristics for choosing the decision bound in a "first-succeed" manner (i.e., steering it towards minimizing the cost function). For problems of a more numerical nature with many solutions, one could also search for the optimal solution with binary search instead of decreasing the objective one by one.

Pre- and in-processing, arithmetic. For some problems currently too many cuts are discarded because of coefficients larger than $2^{30}$. One can look for solutions from the implementation point of view, e.g., by using large integer arithmetic, or using floating point arithmetic, but it might also be the case that more constraint simplification preand in-processing techniques can be helpful (and not only for this purpose). For propositional SAT, the so-called lemma shortening techniques introduced in MiniSAT [12] have turned out to be essential for modern SAT solvers and (in fact, several extensions of them) can be applied to IntSat as well. Modern SAT solvers such as lingeling [3] heavily apply different inprocessing techniques to keep the constraint database small but strong.

MIPs. Finally, it needs to be worked out how to apply IntSat in order to solve MIP instances, i.e., where not all variables are subject to integrality. One can decide on the 
integer variables as it is done now, and at any desired point one can run an LP solver to optimize the values for the rational variables. The inclusion of lower bounding techniques, well-known from modern MIP solvers, also needs to be considered.

\section{Experiments}

All experiments described in this section were carried out on a standard $2.66 \mathrm{GHz} 4$-core Intel i5 750 desktop. The reader can easily verify these results; in particular our prototype IntSat implementation including source code and all benchmarks can be downloaded from [19].

CPLEX and Gurobi. We compare with the newest versions of the commercial solvers CPLEX (v.12.6) and Gurobi (v.5.6.2). Both use all four processor cores (while IntSat uses only one!). Both are well-known to outperform, in general by far, the existing noncommercial solvers. The technology behind these solvers is extremely mature, after decades of improvements: according to [5], between 1991 and 2012 they have seen a 475000 times speedup from algorithmic improvements only (i.e., not counting another 2000 times from hardware improvements)!.

Rather than using a single method, these solvers apply a large variety of techniques, including, e.g., specialized cuts (Gomory, knapsack, flow and GUB covers, MIR, clique, zerohalf, mod-k, network, submip,etc.), heuristics (rounding, RINS, solution improvement, feasibility pump, diving, etc.) and variable selection techniques (pseudo costs, strong branching, reliability branching, etc.).

They also apply sophisticated presolve methods to reduce in advance the size of the problem and to tighten its formulation. Since we have no special-purpose presolve implementation for IntSat (yet), unfortunately here we had to use Gurobi's one, and for fairness, we used Gurobi to presolve and output all instances (which took essentially negligible time) and ran all three solvers on these Gurobi-presolved instances.

Other classes of solvers. It is well known that SAT Modulo Theories (SMT) [20] solvers such as Mathsat5 [8], Yices [11], Z3 [10] or our own Barcelogic solver [6] mostly focus on efficiently handling the arbitrary Boolean structure on top of the LIA constraints. Their Theory Solver component, the one that handles conjunctions of constraints (our aim here), is rather basic, and we do not compere here with SMT solvers since on conjunctive problems they are indeed in general orders of magnitude worse than CPLEX or Gurobi.

Concerning SAT and Lazy Clause Generation (LCG) [21], from our own work (see among many others [1]), we also know too well that solvers that (lazily) encode linear constraints into SAT can be competitive as long as problems are rather Boolean, without a heavy ILP/optimization component. Also CSP solvers such as Sugar [24], which heavily focus on their rich constraint language, are in general very far from the commercial OR solvers on the typical hard pure ILP optimization problems.

Also, most of these SAT/SMT/LCG solvers cannot optimize or are rather bad at it. Cutsat [14] cannot optimize either. 
Random optimization instances. We used a random generator to create 100 optimization instances with 600 variables (about half of them non-binary) and 750 constraints (instances and generator are available at [19]). Then we discarded the 51 "too easy" instances (for which all three solvers could find an optimal solution and prove optimality in less than $2 \mathrm{~s}$ ).

The first columns (I, C, G) in the table below show runtimes in seconds of, respectively, IntSat, CPLEX and Gurobi to prove optimality, and no time indicates timeout after 10s, which happens 17 times for IntSat, 19 times for CPLEX and 9 times for Gurobi.

Since finding good solutions quickly is perhaps as important as proving optimality, the following columns show the cost of the optimal solution ("opt"), and the best solutions found after $10 \mathrm{~s}$, only when different from the optimal one. The reader can check that IntSat fails 8 times to find the optimal solution, CPLEX 13 times, and Gurobi 7 times, and that the total sum of distances to the optimal solutions in these cases are 22,39 and 17, respectively. When given longer runtimes, the commercial solvers tend to behave better on these instances than the current version of IntSat. However, this should of course be re-evaluated after a more mature implementation, heuristics, and pre- and inprocessing, etc., become available for IntSat.

\begin{tabular}{|c|c|c|c|c|c|c|c|}
\hline & I & $\mathrm{C}$ & $\mathrm{G}$ & opt & I & $\mathrm{C}$ & $\mathrm{G}$ \\
\hline 01.lp & & & & -7 & -5 & . & -4 \\
\hline $.1 p$ & & 7.76 & 5.41 & -7 & . & & \\
\hline 5.lp & 1.85 & 1.08 & 4.19 & -4 & & & \\
\hline 06.lp & 2.50 & & & -13 & . & -7 & 10 \\
\hline 07.lp & 1.06 & 2.98 & 3.94 & -7 & & & \\
\hline 10.lp & 4.21 & 0.17 & 0.07 & -11 & & & \\
\hline 12.lp & & & 9.80 & -9 & & & \\
\hline 14.lp & 0.77 & 5.78 & 3.16 & -14 & & & \\
\hline 15.lp & 1.43 & 2.56 & 0.21 & -10 & & & \\
\hline 16.lp & & & 5.02 & -9 & & -8 & \\
\hline 20.lp & & & & -8 & -6 & -4 & -6 \\
\hline 21.lp & & 0.71 & 0.19 & -7 & -5 & & \\
\hline 23.lp & & & & -8 & & & \\
\hline 24.lp & 2.85 & 0.08 & 0.05 & -4 & & & \\
\hline 26.lp & 1.04 & & 2.93 & -11 & $\cdot$ & -7 & \\
\hline 27.lp & & 2.71 & 6.32 & -2 & & & \\
\hline 28.lp & 2.16 & & & -9 & & -7 & -8 \\
\hline 31.lp & & & & -6 & & -3 & -3 \\
\hline 33.lp & 2.79 & 6.38 & 2.92 & -6 & & & \\
\hline 34.lp & 2.62 & 1.86 & 0.30 & -13 & & & \\
\hline 36.lp & 6.58 & 1.53 & 5.23 & -9 & & & \\
\hline 40.lp & 2.02 & 3.10 & 0.05 & -18 & & & \\
\hline 44.lp & 4.76 & 7.47 & 8.54 & -10 & & & \\
\hline 49.lp & 2.77 & 0.47 & 0.09 & -8 & & & \\
\hline 50.lp & & & 0.22 & -12 & -8 & -11 & \\
\hline
\end{tabular}

\begin{tabular}{|l|rrr|r|rrr|}
\hline & I & C & G & opt & I & C & G \\
\hline 53.lp & & 2.47 & 3.05 & -13 & -7 &. &. \\
$60.1 p$ & 1.69 & & 2.19 & -9 &. & -8 &. \\
$61.1 p$ & 1.47 & 1.14 & & -16 &. &. & -15 \\
$62.1 p$ & 2.58 & 0.53 & 0.02 & -3 &. &. &. \\
$63.1 p$ & & & 4.55 & -12 &. &. &. \\
$64.1 p$ & 1.56 & 6.33 & 2.60 & -4 &. &. &. \\
$65.1 p$ & 3.26 & 0.80 & 0.81 & -8 &. &. &. \\
$66.1 p$ & 1.32 & 9.23 & 4.47 & -5 &. &. &. \\
$68.1 p$ & & & & -9 &. &. &. \\
$69.1 p$ & 5.91 & 1.20 & 0.13 & -14 &. &. &. \\
$70.1 p$ & 8.33 & 0.24 & 0.09 & -6 &. &. &. \\
$73.1 p$ & & & 4.75 & -11 & -9 & -9 &. \\
$76.1 p$ & 0.74 & 2.89 & 0.40 & -11 &. &. &. \\
$78.1 p$ & & & & -8 & -5 & -2 & -4 \\
$79.1 p$ & 8.26 & 0.36 & 0.06 & -4 &. &. &. \\
80.lp & 7.54 & 2.59 & 0.13 & -7 &. &. &. \\
$81.1 p$ & 0.86 & 4.71 & 4.78 & -9 &. &. &. \\
$84.1 p$ & 2.93 & & 6.40 & -12 &. & -5 &. \\
$87.1 p$ & & & & -10 &. & -8 &. \\
$88.1 p$ & & & 9.67 & -5 & -4 & -4 &. \\
$91.1 p$ & 2.38 & & 2.96 & -9 &. &. &. \\
$93.1 p$ & 2.98 & 3.03 & 0.45 & -9 &. &. &. \\
$95.1 p$ & 1.51 & 3.13 & 0.14 & -10 &. &. &. \\
$99.1 p$ & 0.62 & 2.00 & 4.92 & -7 &. &. &. \\
\hline
\end{tabular}




\subsection{MIPLIB instances}

From the MIPLIB 2010 Mixed Integer Problem Library, a well-known "standard test set" to compare optimizer performance for the Operations Research (OR) community, cf. miplib.zib.de, we considered all 30 ILP instances (i.e., with integer and binary variables only) and discarded the 11 instances lacking initial lower and upper bounds for some variable.

For the remaining 19 ones, the next table below indicates runtimes (in s) needed to prove feasibility (as recommended for the commercial solvers, the objective function was replaced by 0 ). The table also includes some statistics on number of constraints, total number of variables, and among these, the number of binary variables.

We ran IntSat with no presolving, as Gurobi's presolve was harmful for it in some cases. Here we also compare with the Cutsat implementation of [14], which currently can only handle feasibility, and no optimization.

\begin{tabular}{l|rrrr|rrr|} 
& \multicolumn{5}{|c|}{ Feasibility (s) } & \multicolumn{3}{c|}{ Problem statistics } \\
& IntSat & CPLEX & Gurobi & Cutsat & \#constr. & total \#vars. \#0/1-vars. \\
\hline 30n20b8 & 20.14 & 0.83 & 0.41 & $>300$ & 666 & 18380 & 11036 \\
d10200 & 10.00 & 0.20 & 0.22 & $>300$ & 1147 & 2000 & 733 \\
d20200 & 0.55 & 0.34 & 1.15 & $>300$ & 1702 & 4000 & 3181 \\
lectsched-1 & 1.11 & 7.75 & 39.73 & 64.84 & 51608 & 28718 & 28236 \\
lectsched-1-obj & 1.09 & 145.25 & 11.71 & 45.77 & 51608 & 28718 & 28236 \\
lectsched-2 & 0.60 & 3.50 & 1.14 & 5.39 & 31775 & 17656 & 17287 \\
lectsched-3 & 0.99 & 6.94 & 6.82 & 18.43 & 46615 & 25776 & 25319 \\
lectsched-4-obj & 0.25 & 0.15 & 0.38 & 0.86 & 14760 & 7901 & 7665 \\
mzzv11 & 1.27 & 0.04 & 1.26 & 0.08 & 12871 & 10240 & 9989 \\
neos-1224597 & 0.20 & 0.11 & 0.15 & $>300$ & 3682 & 3605 & 3150 \\
neos16 & 0.09 & 9.74 & 16.54 & $>300$ & 1028 & 377 & 336 \\
neos-555424 & 0.07 & 0.07 & 0.07 & 18.53 & 2746 & 3815 & 3800 \\
neos-686190 & 1.98 & 0.44 & 0.27 & $>300$ & 3785 & 3660 & 3600 \\
ns1854840 & 3.21 & 3.31 & 1.57 & 3.75 & 151216 & 135754 & 135280 \\
rococoB10-011000 & 0.06 & 0.05 & 0.06 & 0.06 & 3063 & 4456 & 4320 \\
rococoC10-001000 & 0.05 & 0.04 & 0.05 & 4.45 & 2298 & 3117 & 2993 \\
rococoC11-011100 & 0.10 & 0.16 & 0.10 & 0.05 & 4403 & 6491 & 6325 \\
rococoC12-111000 & 0.16 & 0.15 & 0.27 & $>300$ & 13181 & 8619 & 8432 \\
sp98ir & 0.16 & 0.15 & 0.28 & 0.92 & 1531 & 1680 & 871 \\
\hline
\end{tabular}

\subsection{Optimizing the MIPLIB instances}

We also considered optimizing these same 16 MIPLIB instances (lectsched-1, -2 and -3 are feasibility ones), using all three applicable solvers (IntSat,CPLEX,Gurobi) with a timelimit of $600 \mathrm{~s}$.

30n20b8 and lectsched-4-obj: For these two instances, all three solvers find the optimal solution and prove optimality in less than 10 seconds. For $30 \mathrm{n} 20 \mathrm{~b} 8$, in $1.03 \mathrm{~s}, 3.59 \mathrm{~s}$ and 4.18s, respectively for IntSat, CPLEX, and Gurobi (optimal solution has cost 302) and for lectsched-4-obj, in $0.43 \mathrm{~s}, 3.37 \mathrm{~s}$, and $1.61 \mathrm{~s}$ respectively (optimal is 4 ). Note that 
on both instances IntSat is fastest. IntSat is currently not able to prove optimality for any of the other 14 MIPLIB optimization instances in less than 600s.

neos16 and neos-1224597: IntSat does find optimal solutions quickly for these two problems. For neos 16, none of the three solvers proves optimality in less than 600s, but IntSat finds the optimal solution (cost 446) in 13.1s, whereas CPLEX needs 39.2s and Gurobi needs 45s. For neos-1224597, all three solvers find the optimal solution (cost -448) in around 1s., but CPLEX and Gurobi moreover prove optimality.

The other 12 instances: CPLEX and Gurobi also prove optimality for five other instances, the same ones for both solvers, given in the first table below.

Results and best found solutions for the remaining seven instanes are given in the second table below. Out of these seven, two (d10200, rococoC11) are catalogized in the MIPLIB as "hard" and three further instances (d20200, lectsched-1-obj, and ns 1854840) are "open", as their optimal cost is unknown. IntSat is the best solver by far on the open problem ns 1854840, even though IntSat's 600s refer to runtime on one core only, whereas the other solvers run 600s on all four cores. In fact, for this instance Gurobi only finds an initial "heuristic" solution that is more than 100 times worse than the one found by IntSat; this happens because Gurobi's root simplex times out after 600s. Of the other "open" problems, IntSat is also better than CPLEX on two other problems: lectsched-1-obj and rococoC11-011100.

In some cases IntSat appears to be quite improvable still, e.g., due to its too naive handling of very large input problems. Sometimes also better optimization heuristics will to be useful on instances with a very numerical nature and slowly decreasing values of the objective function.

\begin{tabular}{l|rr|rr} 
& \multicolumn{3}{|c|}{ Best solutions found } & \\
& IntSat (600s) & optimal & time CPLEX & \\
\hline mzzv11 & -18368 & -21718 & $16.64 \mathrm{~s}$ & $21.73 \mathrm{~s}$ \\
neos-555424 & 1369300 & 1324300 & $4.72 \mathrm{~s}$ & $2.16 \mathrm{~s}$ \\
neos-686190 & 11380 & 6730 & $28.95 \mathrm{~s}$ & $24.21 \mathrm{~s}$ \\
rococoC10-001000 & 13402 & 11460 & $49.89 \mathrm{~s}$ & $436.31 \mathrm{~s}$ \\
sp98ir & 279007104 & 219676790 & $24.17 \mathrm{~s}$ & $33.07 \mathrm{~s}$ \\
\hline
\end{tabular}

\begin{tabular}{l|rrr|} 
& \multicolumn{3}{|c}{ Best solutions found after 600s } \\
& IntSat & CPLEX & Gurobi \\
\hline $\mathrm{d} 10200$ & 12809 & 12441 & 12438 \\
$\mathrm{~d} 20200$ & 13619 & 12279 & 12262 \\
lectsched-1-obj & 92 & 93 & 85 \\
ns1854840 & 288000 & 392000 & 4272000 \\
rococoB10-011000 & 21462 & 19449 & 19810 \\
rococoC11-011100 & 21427 & 21800 & 20957 \\
rococoC12-111000 & 57118 & 36988 & 35845 \\
\hline
\end{tabular}




\section{Related Work and Conclusions}

We already mentioned the work on Cutsat [14]. The idea of applying conflicting sets is not only reminiscent to the conflict analysis of SAT, but also of SAT Modulo Theories (SMT) [20,2] for the theory of linear arithmetic, with the main difference, among others, that here new ILP constraints are obtained by cut inferences, normalized and learned, and not only new Boolean clauses that are disjunctions of literals representing bounds (usually only those that occur in the input formula). Other SAT/SMT related work, but for rational arithmetic is $[17,15,9]$.

It is also worth mentioning that there may be some possible theoretical and practical consequences of the fact that IntSat's underlying cutting planes proof system is stronger than CDCL's resolution proof system: could IntSat outperform SAT solvers on certain SAT problems for which no short resolution proofs exist? E.g., pigeon-hole-like situations do occur in practical problems (think of timetabling or scheduling). A similar question applies to the current SMT solvers, which are based on resolution as well [18].

It seems unlikely that for ILP or MIP solving one single technique can dominate the others; the best solvers will probably continue combining different methods from a large toolbox, which perhaps will also include IntSat at some point. Still, IntSat by itself already appears to be the first alternative method for ILP that uses no LP relaxations and no simplex that is competitive on certain hard optimization problems, and moreover it still has an enormous potential for enhancement. We expect that this work will trigger quite some further activity on all the improvements mentioned in Section 4.

Acknowledgments. Thanks go to Albert Fiol for his work last year, as an undergraduate student, on a preliminary IntSat implementation, to Enric Rodríguez and Albert Oliveras for always trying to answer my questions, and to Dejan Jovanović for his help with cutsat and to my muse Mariona. Partially supported by the Spanish government under the SweetLogics project (TIN 2010-21062-C02-01).

\section{References}

1. Ignasi Abío, Robert Nieuwenhuis, Albert Oliveras, Enric Rodríguez-Carbonell, and Peter J. Stuckey. To Encode or to Propagate? The Best Choice for Each Constraint in SAT. In 19th International Conference on Principles and Practice of Constraint Programming, CP'13, pages 97-106. Springer Berlin Heidelberg, 2013.

2. Clark W. Barrett, Roberto Sebastiani, Sanjit A. Seshia, and Cesare Tinelli. Satisfiability modulo theories. In Handbook of Satisfiability, pages 825-885. IOS Press, 2009.

3. Armin Biere, 2010. Lingeling SAT Solver. Available at http://fmv . jku . at/lingeling/.

4. Armin Biere, Marijn J. H. Heule, Hans van Maaren, and Toby Walsh, editors. Handbook of Satisfiability, volume 185 of Frontiers in Artificial Intelligence and Applications. IOS Press, February 2009.

5. Bob Bixby. Presentation: 1000X MIP Tricks, 12 June 2012, Bill Cunninghams 65th, 2012.

6. Miquel Bofill, Robert Nieuwenhuis, Albert Oliveras, Enric Rodríguez-Carbonell, and Albert Rubio. The Barcelogic SMT Solver. In Computer-aided Verification (CAV), volume 5123 of Lecture Notes in Computer Science, pages 294-298, 2008. 
7. Vasek Chvátal. Edmonds polytopes and a hierarchy of combinatorial problems. Discrete Mathematics, 4(4):305-337, 1973.

8. Alessandro Cimatti, Alberto Griggio, Bastiaan Schaafsma, and Roberto Sebastiani. The MathSAT5 SMT Solver. In Nir Piterman and Scott Smolka, editors, Proceedings of TACAS, volume 7795 of $L N C S$. Springer, 2013.

9. Scott Cotton. Natural domain smt: A preliminary assessment. In Formal Modeling and Analysis of Timed Systems - 8th International Conference, FORMATS 2010, pages 77-91. Springer LNCS 6246, 2010.

10. L. de Moura and N. Bjorner. Z3: An Efficient SMT Solver. Technical report, Microsoft Research, Redmond, 2007. Available at http://research.microsoft.com/projects/z3.

11. B. Dutertre and L. de Moura. The YICES SMT Solver. Technical report, Computer Science Laboratory, SRI International, 2006. Available at http://yices.csl. sri . com.

12. Niklas Eén and Niklas Sörensson. An Extensible SAT-solver. In E. Giunchiglia and A. Tacchella, editors, 6th International Conference on Theory and Applications of Satisfiability Testing, SAT '03, volume 2919 of Lecture Notes in Computer Science, pages 502-518. Springer, 2004.

13. Ralph E. Gomory. Outline of an algorithm for integer solutions to linear programs. Bulletin of the American Mathematical Society, 64(5):275-278, 1973.

14. Dejan Jovanovic and Leonardo Mendonça de Moura. Cutting to the chase - solving linear integer arithmetic. J. Autom. Reasoning, 51(1):79-108, 2013.

15. Konstantin Korovin and Andrei Voronkov. Solving systems of linear inequalities by bound propagation. In CADE-23 - 23rd Int. Conf. on Automated Deduction, pages 369-383, 2011.

16. J. Marques-Silva and K. A. Sakallah. GRASP: A Search Algorithm for Propositional Satisfiability. IEEE Transactions on Computers, 48(5):506-521, 1999.

17. Kenneth L. McMillan, Andreas Kuehlmann, and Mooly Sagiv. Generalizing dpll to richer logics. In Computer Aided Verification, 21 st International Conference, CAV, pages 462-476. Springer LNCS 5643, 2009.

18. Robert Nieuwenhuis. SAT and SMT Are Still Resolution: Questions and Challenges (invited talk). In Automated Reasoning - 6th International Joint Conference, IJCAR, pages 10-13. Springer LNCS 7364, 2012.

19. Robert Nieuwenhuis. Intsat source code, makefile, benchmarks and benchmark generators, 2014. www. lsi. upc. edu/ ${ }^{\sim}$ roberto/IntSatCP2014.tgz.

20. Robert Nieuwenhuis, Albert Oliveras, and Cesare Tinelli. Solving SAT and SAT Modulo Theories: From an abstract Davis-Putnam-Logemann-Loveland procedure to DPLL(T). Journal of the ACM, JACM, 53(6):937-977, 2006.

21. Olga Ohrimenko, Peter J. Stuckey, and Michael Codish. Propagation = lazy clause generation. In Christian Bessiere, editor, CP, volume 4741 of Lecture Notes in Computer Science, pages 544-558. Springer, 2007.

22. Knot Pipatsrisawat and Adnan Darwiche. On modern clause-learning satisfiability solvers. Journal of Automated Reasoning, 44(3):277-301, 2010.

23. Alexander Schrijver. Theory of Linear and Integer Programming. John Wiley \& Sons, Chichester, 1986.

24. Naoyuki Tamura, Akiko Taga, Satoshi Kitagawa, and Mutsunori Banbara. Compiling finite linear CSP into SAT. Constraints, 14(2):254-272, 2009. 